\title{
V: Gedächtnissrede auf Karl Schroeder
}

gehalten

\author{
beim Wiederbeginn der Klinik am 14. Februar 1887 \\ von

\section{Hofmeier,}

Secundärarzt der Königlichen Universität-Frauenklinik zu Berlin.

M. H.! Als ich Ihnen heute vor 8 Tagen mittheilte, dass während der kommenden Woche der klinische Unterricht angesichts der bevorstehenden Ereignisse ausfallen miisse, geschah es ganz unter dem Eindruck des trostlosen Anblickes, den wir am Sterbebette unseres theuren Lehrers empfangen hatten. Heute, wo wir zum ersten Mal nach dem traurigen Ereigniss der vergangenen Woche uns wieder hier versammelt haben, wo wir noch tief gebeugt von dem Bewusstsein dessen, was wir verloren, kaum die nöthige Sammlung finden, um in unserm Fach weiter zu lehren und zu lernen, lassen Sie uns absehen von unserem gewöhnlichen Arbeitsstoffe und gestatten Sie mir, Ihnen zur bleibenden Erinnerung an Ihren Lehrer in kurzen Ziigen, soweit ich es vermag, ein Bild seines äusseren Lebensganges und Entwickelungsganges zu entrollen! Lassen Sie uns die erste Stunde des Beisammenseins in diesem Raum, in dem Sie so oft seine lehrenden Worte gehört haben, seinem Andenken widmen!

Wenn ich mich dazu bernfen fühle, Sie hierzu aufzufordern, so geschieht es nicht nur deshalb, weil ich augenblicklich im Auftrage des Königl. Ministeriums die Ehre habe ihn zunächst zu vertreten, sondern weil von allen seinen Schülern ich selbst am längsten das Glück gehabt habe unter ihm und mit ihm an dieser Anstalt zu wirken, und weil ich in fast 10 jährigem persönlichen, täglichen Verkehr nicht nur seine wissenschaftlichen Grundsätze und Anschauungen kennen zu lernen Gelegenheit hatte, sondern auch in ihm neben dem Lehrer den Mensclien, fast möchte ich sagen den Freund, verehren und lieben gelernt habe. Mag es der Zukunft überlassen sein, den Werth des Bleibenden aus seinen Werken zu bezeichnen, mag es beredteren Zungen vorbehalten sein, seine bedeutende Persönlichkeit zu preisen: Niemand, glaube ich, wird in das, was er wollte und erstrebte, besser eingeweiht sein, wie Diejenigen, welche in der ruhmvollsten Dekade seines Lebens ihm helfend zur Seite zu stehen die Ehre und Freude hatten!

Was zunächst den äusseren Lebensgang unseres verstorbenen Meisters anbelangt, so war derselbe zwar einfach, aber immerhin interessant genug, dass wir Manches daraus lernen können, und werth, dass ich Ihnen einige Daten aus demselben mittheile: Geboren am 11. September 1838 in Neu-Strelitz als Sohn des Rectors der dortigen Mädchenschule, besuchte er dort das Gymnasium und bezog nach Absolvirung desselben mit 20 Jahren die Universität Rostock und später Würzburg, wo er nach dem Zeugniss seiner Studiengenossen eines der flottesten, aber auch der fleissigsten Mitglieder des Corps Hasso-Nassovia war. Nach Absolvirung seines Staatsexamens in Rostock wurde er zunächst bei Thierfelder Assistent und wollte sich schon in einer kleinen mecklenburgischen Landstadt als Arzt niederlassen, als er von Prof. Gustav Veit das Anerbieten erhielt, im Jahre 1864 mit ihm als Assistent nach Bonn zu gehen. Ohne viel Besinnen, allerdings auch ohne grössere Kenntnisse in dem neuen Fach, nahm er dieses Anerbieten an unter der Bedingung, dass ihm das damals noch nothwendige preussische Staatsexamen erlassen würde. Denn die Examina liebte er weder als Examinand noch als Examinator. Ich erinnere mich noch sehr lebhaft, wie drastisch er uns eines Abends in seinem Garten zu Pankow vor einigen Jahren seine Verlegenheit schilderte, als er zum ersten Mal in die Poliklinik zu einem Nabelschnurvorfall gerufen wurde. Ohne eine 
klare Idee darüber, was in einem solchen Fall wohl zu thun sei, eilte er zu Veit, um ihm seine Noth zu klagen. Dieser iustruirte ihn, so gut es ging, und zu seiner eignen nicht geringen Ueberraschung entwickelte er ein lebendes Kind. Von dem Eifer und dem Fleiss, welche er während seiner Assistentenzeit entfaltete, zengte auch eine wohl beglaubigte Aeusserung von $\mathrm{V}$ eit dahin gehend, dass er nur zweimal in seiner Anstalt grössere Puer peralfieberepidemien gehabt habe; davon die eine zu Schroeder's Assistentenzeit: denn der habe eben Alles untersuchen wollen. Die Frncht dieses Eifers war seine erste hervorragende wissenschaftliche Arbeit: die Monographie "Schwangerschaft, Geburt und Wochenbett", welche eine grosse Zahl eigener Beobachtungen besonders in Bezug auf die Physiologie enthält. Nach zweijähriger Assistentenzeit habilitirte sich Schroeder in Bonn und las in seinem ersten Semester vor drei Zuhörern. Nach vierjähriger Thätigkeit in Bonn wurde er 1868 nach Erlangen zunächst als Extraordinarius berufen, um Rosshirt zu unterstïtzen ein Jahr später wurde er Ordinarius. Die Beschreibung der Zustände, wie er sie zu jener Zeit in seinem Fach in Erlangen vorfand, klangen oft fast unglaublich. Gynäkologie existirte überhaupt nicht, in seiner Praxis hatte er in dem ersten Jahr 8 gyı̈̈kologische Kranke; auch geburtshilflich war die Thätigkeit sehr gering. Hier fand er Zeit, sein Werk über Schwangerschaft, Geburt und Wochenbett zu einem "Lehrbuch der Geburtshilfe ${ }^{\omega}$ zu elweitern (1870) und fand ferner die Zeit sein Lehrbuch über die "Krankheiten der weiblichen Geschlechtsorgane" herauszugeben, welches als Abtheilung des bekannten Ziemssen'schen Sammelwerkes erschienen ist. Die Erfolge beider Lehrbücher sind Ihnen ja bekannt. Das erste ist im vorigen Sommer in 9. Auflage von 5000 Exemplaren erschienen, von dem zweiten ist eben jetzt die 8. Auflage im Druck. Wesentlich das erstere, welches schon in hervorragender Weise die Merkmale des Schroeder'schen Geistes zeigte: Klarheit und Einfachheit der Sprache und Darstellung, Präcision des Ausdrucks und scharfe Logik, machte seinen Namen bald bekannt und lenkte die allgemeine Anfmerksamkeit auf ihn, so dass bei Erledigung des Berliner Lelırstuhls der Geburtshilfe - es gab zu jener Zeit nur einen - durch den Tod von E. Martin die Berliner Facultät ihn in erster Linie zum Nachfolger vorschlug. Mit der Bescheidenheit, welche ihn bis in seine letzten Lebenstage in Bezug auf seine Person auszeichnete, dachte er damals so wenig daran nach Berlin zu kommen, dass er sich mit der Bitte an den inzwischen ebenfalls verstorbenen und damals vielfach als bestimmten Nachfolger Martin's angeseheneu Spiegelberg wandte, seinen Einfluss in Breslau bei einer eventuellen Vakanz für ihn geltend zu machen: eine Bitte übrigens, welche ihm von diesem Letzteren ziemlich ohne Umschweife abgeschlagen wurde. Allgemein war die Ueberraschung zum Geringsten nicht von Schroeder selbst - als er telegraphisch die Nachricht von seiner Berufung nach Berlin bekam. Am 1. April 1876 trat er seine neue Lehrthätigkeit hier an im Alter von $37 \frac{1}{2}$ Jahren, ein Mann in der Vollkraft seiner Jahre, ausgerüstet mit dem festen Willen und dem Können, Grosses in seinem Fach hier zu schaffen. Das Feld seiner Thätigkeit war damals die alte Entbindungsanstalt Dorotheenstrasse 5 und die gynäkologisehe Abtheilung in der Charité. In der ersteren fungirten neben ihm ausser einem anatomischen drei klinische Assistenten, in der Charité ein Stabsarzt. Als im Jahre 1878 die zweite ordentliche Professur fïr unser Fach hier geschaffen ward, wurde Schroeder der Neubau einer grossen Anstalt vom Ministerium in nahe Aussicht gestellt, welche, Geburtshilfe und Gynäkologie umfassend, allen Anforderungen der Neuzeit entsprechen sollte. Die Schwierigkeit war zunächst hier ein passendes Terrain mitten in der Stadt zu finden. Ich erinnere mich noch, mit welcher Freude er uns eiues Abends erzählte, dass die Kaufcontracte für das Grundstück (1 200000 Mark) endlich abgeschlossen seien. Die Pläne von Gropius und Schmieden wurden nach Schroeder's Ideen angefertigt und auf das Sorgfältigste von ihm selbst revidirt. Seine Geduld wurde auf eine harte Probe gestellt, als sich in Folge äusserer Verhältnisse der Beginn des Baues immer wieder verzögerte. Dann aber wurde derselbe sehr energisch gefördert, so dass endlich am 16. August 1882 der von uns Allen sehnlichst erwartete Tag erschien, an welchem dieses Haus bezogen werden konnte. Die Ideen, welche $\mathrm{S} c \mathrm{hroeder}$ bei dem Bau der Anstalt leiteten und nach denen er selbst den Unterricht in der Anstalt zu leiten gedachte, entwickelte er in einer glänzenden Rede am 18. October 1882, als unter Anwesenheit fast sämmtlicher noch lebenden Assistenten der Anstalt, darunter eine grosse Zahl von ordentlichen Professoren unseres Faches, die offizielle Einweihung der Anstalt erfolgte. Die Zusammenfassung von Geburtshilfe und Gynäkologie in einem Hause, zugleich doch bei möglichster räumlicher Trennung, die Isolirung der Wöchnerinnen, soweit sich dieselbe mit den Zwecken einer grossen Anstalt verträgt, die Construction möglichst aseptischer Entbindungs- und Operationssäle, die räumliche Isolirung der infectiös Erkrankten: das Alles sind Schroeder'sche Gedanken, welche im Bau dieses Hauses zur
Durchführung gekommen sind. "Wenn", so schloss er ungefähr damals seine Rede, in Bezug auf die äussere Erscheinung der Anstalt Viel, Manchem vielleicht zu Viel gethan zu sein scheint, so liegt neben günstigen äusseren Verhältnissen die Ursache hierfür darin, dass ich es für erstrebenswerth hielt, den jungen in die oft sehr prosaische Praxis gehenden Aerzten einen gewissen idealen Eindruck auch in der äusseren Erscheinung der Wissenschaft aus ihrer Studienzeit mit auf den Weg zu geben, der ihnen für's Leben bleibt."

Der innere Dienst in der Anstalt wurde so organisirt, dass neben dem anatomischen zuerst 5 jetzt 6 Assistenten die einzelnen $A b-$ theilungen leiteten. Um daneben auch noch anderen jüngeren Aerzten die Möglichkeit zu bieten, sich besonders gut fïr die Praxis vorzubilden, wurden 4 sogenannte Volontairarztstellen eingerichtet, ausserdem die Möglichkeit geschaffen, die grosse geburtshilfliche Poliklinik von 8 auf 3 Monate im Hause wohnenden Studenten leiten zu lassen, eine Finrichtung, welche meines Wissens nur hier bestelit. Wie grossen Werth Schroeder auf die Ergänzung des klinischen Unterrichts durch den patloologisch-anatomischen legte, wissen Sie Alle. Uns hat er diese Ideen in ausführlicher Weise dargelegt, als wir im April 1886 im engsten Kreise seiner sämmtlichen Assistenten den Tag festlich begingen, an welchem er vor 10 Jahren die Leitung der Anstalt übernahm. Dieser Richtung in seiner Lehrthätigkeit entsprach es auch, dass er seit Jahren einen Lieblingsgedanken verfolgte: mit einem wïrdigen Leichenhause auch eine Art pathologisches Laboratorium in der Anstalt zn schaffen, um so besonders energisch die Forschung nach der Aetiologie der Infectionskrankheiten in die Hand nehmen zu können.

Unüberwindlich erscheinende Schwierigkeiten stellten sich lange Zeit der Ausführung dieses Planes entgegen, dessen Vollendung er leider nicht melır erleben sollte. Das Haus steht zwar fertig da, aber die Einrichtung fehlt noch gänzlich, so dass die Inbetriebsetzung desselben, welche Schroeder seit Jahren so sehnlichst wüuschte, immer noch in gemessene Ferne gerückt erscheint.

Mitten also aus diesen Ideen zur fortwähreuden Erweiterung und Vergrösserung dieser Lehr- und Arbeitsanstalt, welche er sich als eine Art von Centralinstitut für alle Zweige unserer Wissenschaft dachte, hat ihn ein jäher Tod hinweggeraftit. Wenn auch die Krankheit ja wahrscheinlich schon lange in seinem Körper schlummerte, war doch ihr Ausbruch uud Verlauf ein so acuter, dass man kaum recht zur Besinnung gekommen ist. Es ist Ihnen wohl bekannt, dass seine Erkrankung vermuthungsweise auf eine Infection zurückgeführt wird, welche er sich im December 1880 bei der Spaltung eines jauchigen Abscesses am rechten Auge zuzog. Es stellte sich eine heftige, für diphtheritisch erklärte Conjunctivitis ein, welche sich lange hiuzog, und an die sich ein unbestimmter Fieberzustand anschloss, aus dem dann eine heftige, damals als eine linksseitige Pleuropneumonie gedeutete Entzündung hervorging. Die Section hat ergeben, dass es sich damals um einen mediastinalen, in den Oesophagus perforirten und jetzt vollkommen zurückgebildeten Abscess handelte, dessen Zusammenhang mit dem jetzigen rechtsseitigen Hirnabscess ja allerdings nur vermuthet werder kann. Nach mancherlei Symptomen, die jetzt nachträglich bekanut geworden sind. erscheint es wahrscheinlich, dass sich bereits seit einem Jahr der Gehirnabscess langsam entwickelt resp. weiterverbreitet hat und jetzt plötzlich in den rechten Ventrikel durchgebrochen ist. Sein Gesundheitszustand machte ihm und uus schon seit längerer Zeit Sorge, wenngleich er und wir freilich die Gefahr ganz wo anders suchten, als wie sie sich schliesslich herausgestellt hat.

So, m. H., war das äussere Leben unseres theuren Lehrers: aus kleinsten Anfängen zu der grössten Lehr- und praktischen Thätigkeit an der Spitze eines Instituts, welches, Dank der verständnissvollen und allezeit bereiten Förderung durch das Kol Cultusministerium, nicht nur räumlich das grösste und schönste seiner Art in Deutschland ist, sondern unter seiner Leitung als ein Musterinstitut in der ganzen Welt bekannt wurde. Sie wissen es selbst, wie sehr dasselbe in den letzten Jahren immer mehr ein Sammelpunkt auch für Aerzte und Patientinnen aus aller Herren Länder wurde.

Man hat von manchen Seiten versucht Schroeder den Vorwurf zu machen, dass er im Unterricht der jüngeren Aerzte die Geburtshilfe über der Gynäkologie vernachlässige. Wer seinem Unterricht längere Zeit beigewohnt hat, wer besonders näher seine wissenschaftlichen Ueberzeugungen kannte, weiss, wie unbegründet dieser Vorwurf ist, und ich möchte die Gelegenheit nicht vorübergehen lassen, ohne nachdrücklich dagegen zu protestiren. Er wollte nicht nur stets den Hauptnachdruck auf den geburtshilflichen. Unterricht gelegt sehen, er betonte auch immer, dass die Abtrennung der $\mathrm{Gy}$ näkologie von der Geburtshilfe, wie sie in anderen Ländern (England, Frankreich) z. Th. noch existirt, zu einem schlimmen Spezialistenthum führen müsse: dass nur ein guter Geburtshelfer auch ein guter Gynäkolog sein könne. Dass seine gynäkolologische Thätigkeit dabei in den letzten Jahren mehr bekannt ge- 
worden ist, liegt nicht nur daran, dass er an der Spitze der grössten gynäkologischen Klinik stand und im Mittelpunkt einer aussèrordentlich ausgedehnten gynäkologischen Praxis, sondern wesentlich auch in der Art der erreichten Erfolge, die durch ihre Handgreiflichkeit und Neuheit viel von sich reden machten. Einer seiner letzten und schönsten Erfolge derart liegt gerade auf geburtshilflichem Gebiet: Jener am 3. Januar dieses Jahres wegen Uterusmyom von ihm nach Porro ausgeführte Kaiserschnitt, über welchen er Ihnen seiner Zeit referirte. Einer der grössten und schönsten Kränze auf seinem Sarge zeugte von der Verehrung der dankbaren Mutter. Auch seine erste und letzte wissensehaftliche Arbeit liegt auf geburtshilflichem Gebiet; "Schwangerschaft, Geburt und Wochenbett ${ }^{4}$, erschien 1867 und der Atlas „Der schwangere und

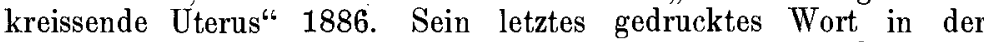
Nummer vom 1. Januar $1887 \mathrm{der}$ von hier aus geleiteten deutschen Hebammenzeitung galt der Reform des Hebammenstandes. Auch ein aus dem vorigen Sommer herrührender Brief eines ihm persönlich befreundeten Ordinarius, welcher mir leider im Original nich wieder vorlag, gab hierfür ein beredtes Zeugniss ab, indem er ihn beglückwünsclite dazu, dass er mit seiner neuesten Arbeit an seine ersten bedeutenden wissenschaftlichen Arbeiten wieder anknüpfe, nachdem er der deutschen Gynäkologie die operativen Wege geebnet.

Eine Kritik seiner wissenschaftlichen Arbeiten geben zu wollen kann mir nicht anstehen, besonders da ich selbst wenigstens in den letzten Jahren vielfach das Glück hatte daran Theil zu nehmen. Ich habe Ihnen nur, m. H., einen kurzen Umriss seines äusseren Entwicklungsganges und seines Strebens geben wollen, zugleich als eine Mahnung und einen Fingerzeig für uns Alle, wie allein man im Leben Grosses erreichen kann. Denn ziehen wir die Quintessenz aus diesem Leben, so ist es Arbeit gewesen, und wieder Arbeit! Seine unbezwingliche Arbeitslust und Freudigkeit kounte nicht schöner gezeichnet werden als in dem Citat in seiner Leichenrede: „Verbiete du dem Seidenwurm zu spinnen, wemn er siclı schon dem Tode näher spinnt!" Vom Morgen bis zum Abend arbeitend, riss er auch den Nichtwollenden mit sich fort, wie wir es oft genug an uns selber Alle erfahren haben, bis er uns selbst einen Funken von seiner eigenen Arbeitslust eingehaucht hatte. Mit wahrhaft jugendlichem Feuer, um das wir ihn oft beneidet haben, stürzte er sich auf eine neue Arbeit. Kein Gang war ihm zu viel, keine Zeit zu unbequem, wenn es seine Arbeit galt! Alle mussten mit! Dabei entsprach es seinem thätigen, energischen Charakter, wenn diese Arbeit sich mehr auf praktische, wie theoretische Dinge erstreckte. Von den Arbeiten an seiner Klinik legen ausser manchen anderswo erschienenen Abhandlungen die 13 Bände der Zeitschrift für Geburtshilfe und Gynäkologie, die mit seinem Herkommen hier neu in's Leben trat, hinreichend Zeugniss ab.

Nehmen Sie, m. H., zu diesem Ihnen so entworfenen Bilde das hinzu, wie es Ihnen noch vor Augen steht: seine einfache Würde, sein jedem äusserlichen Schein so abholdes Wesen, seine biedere Geradheit und seinen sittlichen Ernst, der jede Unwahrheit hasste, seinen scharfen Verstand, der stets das Wesentliche aus den Dingen herausfand, durch die Nichtbeachtung des Unwesentlichen allerdings auch manchmal verletzen konnte, zusammen das Alles mit einer ungewöhnlichen und oft unerwarteten Menschenkenutniss, so werden Sie mir, wie ich hoffe, darin beipflichten, wenn ich sage: ihm gebührt das schönste, ungeschmälerte Lob, das einem Manne zu Theil werden kann: er war ein ganzer Mann!

Uns aber, seinen Mitarbeitern und Schülern, die wir nur wünschen können, dass das von unserem theuren Lehrer so gross gestaltete Werk in ungeschmälerter Weise weiter bestehe und sich weiter entwickle, uns bleibt nur übrig zu wünschen, dass er einen Nachfolger finden möge, von dem man einst mit demselben Recht und derselben Aufrichtigkeit sagen kann, wie von unserem Meister: Er war ein ganzer Mann! Ehre seinem Andenken!

VI. Chr. Bäumler. Syphilis. Dritte umgearbeitete Aufl v. Ziemssen's Hdb. d. Spec. Path. und Ther. Dritter Band. Chronische Infectionskrankheiten. Erster Theil. Leipzig, F. C. W. Vogel, 1886. Ref. Jos eph.

Als ein untrügliches Zeichen für die Werthschätzung, welche man der Bearbeitung der Syphilis durch Prof. Bäumler in dem Zi em s s e n'schen Handbuche der Spec. Pathol. und Ther. angedeihen lässt, ist es aufzufassen, dass deren dritte Auflage bereits nothwendig geworden ist.

Die genügend bekannten Vorzüge dieser Arbeit lassen sich vielleicht kurz dahin zusammenfassen, dass es ein klinisches Lehrbuch im besten Sinne des Wortes darstellt, in welchem neben der sorgfältigsten Berücksichtigung der Literatur bis auf die neueste Zeit auch der praktische Standpunkt gewahrt und nach dieser Richtung manche Fingerzeige gegeben werden.

Wir können die Lectüre dieses Buches nur angelegentlichst empfehlen, und auch der praktische Arzt, dem wenig Zeit zur Ver- fügung steht, wird dasselbe nicht aus der Hand legen, ohne sich in wissenschaftlicher wie praktischer Hinsicht manche Anregung verschafft zu haben.

Von dem reichen Inhalte sei es uns gestattet, hier nur auf Einiges hinzuweisen:

Als ein unabweisliches Desiderat der nächsten Forschungen auf dem Gebiete der Syphilis betont auch B. die Auffindung des Syphilisbacillus; den Lustgarten'schen Bacillen ist wenigstens für die Secrete jede Bedeutung als diagnostisches Criterium der Syphilis genommen, ihr soustiger Platz wird ihnen erst angewiesen werden können, wenn es gelungen sein wird, Reinkulturen dieser Bacillen zu züchten, ihre Eigenschaften an solchen zu studiren und mit denen anderer Bacterien zu vergleichen.

Ueber die praktisch sehr wichtige Frage der Impfsyphilis giebt B. sein Urtheil dahin ab, dass die Syphilis bei der Vaccination übertragen werden kann. Bekannt ist das Beispiel der Luise Manzoni, welche nicht syphilitisch war, als sie geimpft wurde, und von welcher trotzdem unter 17 von ihr Geimpften auf 7 Syphilis übertragen wurde. Daher kann nicht genug betont werden, dass die Impfung mit animaler Lymphe an Stelle einer solchen mit humanisirter Lymphe treten solle.

Die Möglichkeit einer zweiten Infection, welche im allgemeinen milder zu sein scheint, namentlich je frülier sie nach der ersten eintritt, bejaht B. ebenso wie die damit eng zusammenhängende Frage, ob Syphilis heilbar sei. Wir wissen jetzt mit Bestimmtheit, dass die Syphilis vollständig heilen kann. $\mathrm{Zu}$ bedauern ist nur, dass wir kein sicheres Criterium hierfür besitzen.

Bäumler unterseheidet das Schankercontagium von dem syphilitischen Gifte. Das erstere ist ein pathologisches Agens für sich und zwar weit weniger beständig und gleichmässig, als das syphilitische Gift. $O b$ es sich hierbei um einen ganz bestimmten Mikroorganismus handelt, oder ob mehreren der septisch wirkenden Mikrobien die Fähigkeit zukomme, in das Blut eingeimpft. Schankergeschwüre zu erzeugen, muss erst die Zukunft lehren.

Die Auffassung Bäumler's, dass durch die Infection zunächst ein Localheerd geschaffen werde, in dem sich allmählich das Gift vermehre, und von welchem aus dann hauptsächlich auf dem Wege der Lymphgefässe, aber auch durch das Blut, eine langsame Infection der ganzen Säftemasse eingeleitet werde, giebt für das therapeutische Handeln eine ganz genaue Directive. Sobald eine örtliche Läsion sich unzweifelhaft als ein syphilitischer Primäraffect documentirt, ist eine mercurielle Behandlung einzuleiten.

VII. S. Laskowski. L'embaumement, la conservation des siujets et les préparations anatomiques. Genève-Bâle-Lyon, H. Georg, 1886. 154 S. $8^{0}$. Ref. K. Bardeleben.

Diese, von der Academie des sciences in Caen preisgekrönte Arbeit des Genfer Anatomen dürfte, ausser für die Fachgenossen des Verf., vielleicht für Aerzte, welche mal in die Verlegenheit kommen sollten, eine "Einbalsamirung ${ }^{4}$ oder dergleichen vorzunehmen, von lnteresse sein. Wenn übrigens Verf. sich dem Wick ersheimer'schen Verfahren gegenüber sehr abfällig äussert und hervorhebt, dass es nicht neu sei, obwohl man in Deutschland viel Geschrei darüber gemacht habe ete. etc, so möchte Ref. dem Verf. gegenüber darauf hinweisen, dass auch sein Verfahren - Injection von Glycerin und Carbolsäure - durchaus nicht mehr den Reiz der Neuheit für deutsche Anatomen hat. Ref. weiss genau, dass, ohne "Jahre lange mühsame Untersuchungen" in München, Leipzig und Jena, wahrscheinlich auch anderswo, schon seit dem Beginn der siebzjger Jahre die Leichen mit Glycerin, Carbol und Alkohol injicirt worden, ohne dass darum irgend ein Geschrei oder Geräusch gemacht worden ist. Der Gedanke, Carbol anch gegen Le i c h e n fänlniss zu verwenden, lag z. B. für jemand, der den Feldzug gegen Frankreich als Arzt mitgemacht hatte, sehr nahe. Preiskrönung solcher Gedanken ist allerdings in Deutschland nicht üblich. Der Fall Wickersheimer bildet doch eine fast einzig dastehende Ausnahme. Verf. hat übrigens - da sein Incognito sich nicht wahren liess keine Geldprämie, sodern ein Ehrendiplom erhalten und ist correspondirendes Mitglied der oben genannten Akademie geworden.

VIII. Verhandlungen der Deutschen Gesellschaft für Gynaekologie. Erster Congress, abgehalten zu München vorn 17. bis 19. Juni 1886. Im Auftrage des Congresses herausgegeben von Dr. F. Winckel und Dr. R. Frommel. Leipzig, Breitkopf und Haertel, 1886. Ref. Bokelmann-Berlin.

Das litterarische Ergebniss der Verhandlungen des ersten Congresses der neuentstandenen "Deutschen Gesellschaft für Gynaekologie" liegt uns als 'stattlicher 350 Seiten starker Band vor.

Das im Auftrage des Congresses von dem ersten Vorsitzenden und zweiten Schriftführer, den Herren Winckel und Frommel, herausgegebene Werk führt an seiner Spitze, nächst der Einladung 
zur Theilnahme, das Verzeichniss der zur Gesellschaft gehörigen 68 Mitglieder, unter welchen die namhafteren Gynaekologen Deutschlands fast vollständig, daneben einzelne Vertreter unserer Nachbarländer, sowie Amerikas zu finden sind.

Es folgt die Einzeichnungsliste von 73 Congresstheilnehmern, darunter wiederum eine Reihe klangvoller Namen - wenn wir auch einige der klangvollsten vermissen -, die wohl zur Genüge beweist, dass der Gedanke der Gründung einer Gesellschaft, welche, analog derjenigen für Chirurgie, die Gynaekologen Deutschlands, zunächst in jedem zweiten Jahre, zusammenführen sollte, auf keinen unfruchtbaren Boden fallen konnte.

Dass dies in der That der Fall ist, beweisen nicht minder Zahl und Inhalt der auf die Eröffnungsrede des Vorsitzenden folgenden Vorträge, welche den eigentlich wissenschaftlichen Inhalt des Werkes bilden, und mitsammt den unter regster Betheiligung vor sich gehenden Discussionen fast ausnahmslos in extenso wiedergegeben sind. Die Vorträge, im Ganzen 33 an der Zahl, sind genau in der Reihenfolge, in welcher sie gehalten wurden, aufgeführt und geben daher ein anschaulich trenes Bild der Verhandlungen, wie sie wirklich stattfanden, zu welcher Klarheit der Anschauung ausserdem die Gewissenhaftigkeit, mit welcher die jeden Vortrag und jede Discussion eröffnenden und schliessenden Worte des Vorsitzenden jedesmal angeführt sind, wesentlich beitragen mag.

Form und Ausstattung des Buches lassen nach keiner Seite zu wünschen übrig. Dasselbe wird für alle Theilnehmer des Congresses ein schätzbares Andenken bilden, und denen, welche fern bleiben mussten, die Verhandlungen des ersten Congresses Deutscher Gynaekologen in anschaulichster Form zur Kenntniss bringen. 